\title{
STABILITY OF MINIMAL ORBITS ${ }^{1}$ \\ BY
}

\author{
JOHN E. BROTHERS
}

\begin{abstract}
Let $G$ be a compact Lie group of isometries of a riemannian manifold $M$. It is well known that the minimal principal orbits are those on which the volume function $\mathbf{v}$, which assigns to $p \in M$ the volume of the orbit of $p$, is critical. It is shown that stability of a minimal orbit on which the hessian of $\mathbf{v}$ is nonnegative is determined by the degree of involutivity of the distribution of normal planes to the orbits. Specifically, if the lengths of the tangential components of Lie brackets of pairs of orthonormal normal vector fields are sufficiently small relative to the hessian of $\mathbf{v}$, then the minimal orbit is stable, and conversely. Computable lower bounds are obtained for the values of these parameters at which stability turns to instability. These lower bounds are positive even in the case where $\mathbf{v}$ is constant, and are finite unless the normal distribution is involutive. Several examples in which $M$ is a compact classical Lie group and $G$ is a subgroup of $M$ are discussed, showing in particular that the above estimates are sharp.
\end{abstract}

1. Introduction. Let $G$ be a compact, connected Lie group of isometries of a connected riemannian manifold $M$. Let $\mathbf{v}$ denote the function which assigns to each $p \in M$ the volume of the orbit of $p$. It is well known that the minimal principal orbits of $G$ are precisely those on which $\mathbf{v}$ is critical. Since its discovery in $[\mathbf{H}]$, this result has been highly useful in the study of minimal and area minimizing submanifolds; see, for example, [HL].

An obvious question which arises is whether such minimal orbits are stable, where by stability we mean nonnegative second variation of area. An appealing conjecture is that stable minimal orbits correspond to local minima of $\mathbf{v}$. Such a condition is clearly necessary for stability and is, in fact, sufficient for orbits of codimension one as was shown in [BJ1]. Consider, for example, the circles of latitude on a surface of revolution in $\mathbf{R}^{3}$. On the other hand, for orbits of codimension greater than one this condition is far from sufficient as is shown by the following example: Let $M=\mathbf{S}^{3}$ $\subset \mathbf{C}^{2}$ and $G=\mathbf{S}^{1} \subset \mathbf{C}$ act on $M$ by scalar multiplication. Then all orbits of $G$ are great circles of length $2 \pi$, and none is stable. Although one might reason that this example is pathological bescause the critical points of $\mathbf{v}$ are degenerate, hence conjecture that a hypothesis of strict relative minimum, or even of positive definite hessian (in normal directions to the minimal orbit) might be sufficient to ensure stability, it turns out that even this is not true. One would also like to have a theory

Received by the editors June 7, 1983 and, in revised form, February 27, 1985.

1980 Mathematics Subject Classification. Primary 53C42; Secondary 49F22.

Key words and phrases. Minimal submanifold, stable minimal submanifold, stability, orbit, second variation of area.

${ }^{1}$ This work was partially supported by the National Science Foundation. 
strong enough to treat the case where $M$ is a Lie group with a bi-invariant metric and $G$ is a subgroup acting on $M$ by left multiplication, so that $\mathbf{v}$ is constant, as in the above example with $M=\mathbf{S}^{3}, G=\mathbf{S}^{1}$. As evidence that such orbits can be stable one has the standard embeddings of $\mathbf{S U}(2)$ in $\mathbf{S U}(n)$ and of $\mathbf{S O}(3)$ in $\mathbf{S O}(4)$. Each of these subgroups is of least area in its integral homology class [T].

Using a standard differential form argument one can show that if the distribution of normal planes to the orbits is involutive, then a minimal orbit minimizes area in its integral homology class, and hence is stable. (Here all orbits are assumed to be principal.) In particular, this implies stability of codimension one orbits. Taken together with evidence from David Bindschadler's discussion of the invariant Plateau problem in the case where the boundary consists of finitely many orbits [BD], this leads one to conjecture that stability is determined by the degree of involutivity of the normal distribution as measured by the lengths of the tangential components of Lie brackets of pairs of orthonormal normal vector fields. This conjecture turns out to be true. If these parameters are sufficiently small relative to the hessian of $\mathbf{v}$, then the minimal orbit is stable, and conversely. This is proved in 4.9.

In $\S 3$ we develop a formula for the second variation of area of a minimal principal orbit. To our knowledge this formula is new. It involves Lie derivatives instead of covariant derivatives as in the formula of J. Simons [S].

An immediate corollary of the second variation formula is that if the normal distribution is involutive along a minimal orbit $N$, then $N$ is stable if and only if the hessian $H_{\mathbf{v}}$ of $\mathbf{v}$ is nonnegative on $N$ (4.2). In $\S 4$ we also obtain computable lower bounds for the values of the above-mentioned parameters at which stability turns to instability $(4.3,4.5,4.6,4.8)$. These lower bounds are always positive, even for $H_{\mathrm{v}}=0$ in which case we show by example in $\$ 5$ that they are sharp. For the general case of nonvanishing hessian our results are not sharp. Indeed, most do not involve the size of $H_{\mathbf{v}}$. We defer to a later paper [BJ2] the derivation of a priori second variation estimates involving both the size of $H_{\mathrm{v}}$ and the degree of involutivity of the normal distribution.

In $\S 5$ our results are applied to the discussion of various examples showing, in particular, that products of unitary groups (hence in particular a maximal torus of $\mathbf{U}(n))$ are stable in $\mathbf{U}(n)$, that products of certain special unitary groups are stable in $\mathbf{U}(n)$, and that products of special orthogonal groups are stable in $\mathbf{U}(n)$. We also show that a maximal torus of $\mathbf{S U}(n)$ is not stable in $\mathbf{S U}(n)$.

Finally, I would like to thank Jiri Dadok for several very helpful conversations.

2. Preliminaries. In general we use standard notation and terminology. A good basic reference is $[\mathbf{K N}]$.

With $M$ and $G$ as in the Introduction, we will assume that all orbits of $G$ are principal, that is, that the isotropy groups are conjugate. This implies that the quotient $G \backslash M$ is a smooth manifold and that the quotient map $F: M \rightarrow G \backslash M$ is a smooth submersion. Denote the orbit of $p \in M$ by $N_{p}=\{g(p): g \in G\}$ and set $\operatorname{dim} M=m, \operatorname{dim} N_{p}=n$. Let $\mathbf{v}(p)$ be the $n$-dimensional volume of $N_{p}$. Then $\mathbf{v}(p)=\mathscr{H}^{n}\left(N_{p}\right)$, where $\mathscr{H}^{n}$ is the $n$-dimensional Hausdorff measure on $M$. Note that $\mathbf{v}$ is a smooth function. 
T(M) is the tangent bundle of $M$ and $\mathscr{X}(M)$ is the vector space of smooth vector fields on $M$. For each $V \in \mathscr{X}(M), \mathscr{L}_{V}^{k}$ and $\nabla_{V}^{k}$ are, respectively, the Lie and covariant derivatives with respect to $V$ of order $k=1,2, \ldots$, acting on tensor fields of all types.

Denote by $\sigma$ the metric tensor of $M$, extended in the usual way to the bundle $\Lambda_{k}(M)$ of $k$-vectors tangent to $M$ for $k=2,3, \ldots, m$. Where suitable we also use the notation $\sigma(\xi, \eta)=\langle\xi, \eta\rangle,\langle\xi, \xi\rangle^{1 / 2}=\|\xi\|$. The term "vertical (respectively, horizontal) vector field" will be used to denote a vector field $X \in \mathscr{X}(M)$ such that $X(p)$ is tangent (respectively, normal) to $N_{p}$ for each $p \in M$. If $W$ is a horizontal vector field, then $H^{W}(p)$ denotes the mean curvature of $N_{p}$ at $p \in M$ in the direction $W(p)$. Note that if $W$ is $G$-invariant, then $H^{W}$ is constant on each orbit.

If $f \in C^{\infty}(M)$ has a critical point at $p \in M$, then one defines the hessian $H_{f}(p)$ of $f$ at $p$ to be the bilinear function on $\mathbf{T}_{p}(M)$ such that $H_{f}(p)(v, w)=V \circ W(f)(p)$, where $V, W$ are smooth extensions of $v, w \in \mathbf{T}_{p}(M)$ to a neighborhood of $p . H_{f}(p)$ is symmetric and does not depend on the extensions $V, W$. In terms of local coordinates $\left(x^{1}, \ldots, x^{n}\right), H_{f}(p)$ has matrix $\left[\partial^{2} f / \partial x^{i} \partial x^{j}(p)\right]$.

Fix $p \in M$ and let $X$ be a smooth vector field defined in a neighborhood of $N_{p}$. Denote the flow of $X$ by $\phi_{t}$. For $k=1,2$ the $k$ th variation of area of $N_{p}$ with respect to the deformation vector field $X$ is defined by

$$
\delta_{p}^{(k)}(X)=\left.\frac{d^{k}}{d t^{k}} \mathscr{H}^{n}\left(\phi_{t} N_{p}\right)\right|_{t=0} .
$$

Then $N_{p}$ is minimal if $\delta_{p}^{(1)}(X)=0$ for $X \in \mathscr{X}(M)$ and stable if $\delta_{p}^{(2)}(X) \geqslant 0$ for $X \in \mathscr{X}(M)$.

We recall from [L, p. 7] that if $X \in \mathscr{X}(M)$ has horizontal projection $W$, then

$$
\boldsymbol{\delta}_{p}^{(1)}(X)=\boldsymbol{\delta}_{p}^{(1)}(W)=-\int_{N_{p}} H^{W} d \mathscr{H}^{n} .
$$

We also observe that $[\mathbf{S}, 3.2 .2]$ implies that if $N_{p}$ is minimal, then $\delta_{p}^{(2)}(X)$ depends only on the restriction of $X$ to $N_{p}$ and $\delta_{p}^{(2)}(X)=\delta_{p}^{(2)}(W)$.

\section{Second variation.}

3.1. Lemma. Let $W \in \mathscr{X}(M)$ be horizontal and $\xi$ be a smooth $n$-vector field on an open subset $M_{0}$ of $M$ such that $\xi(p)$ is tangent to $N_{p}$ and $\|\xi(p)\|=\mathbf{v}(p)$ for $p \in M_{0}$. Then

$$
\begin{gathered}
\frac{1}{2}\left(\mathscr{L}_{W} \boldsymbol{\sigma}\right)(\xi, \xi)=-\mathbf{v}^{2} H^{W}=\mathbf{v}(W \mathbf{v}) \\
\frac{1}{2}\left(\mathscr{L}_{W}^{2} \boldsymbol{\sigma}\right)(\xi, \xi)=(W \mathbf{v})^{2}+\mathbf{v}\left(W^{2} \mathbf{v}\right)+\left\langle\mathscr{L}_{W}^{2} \xi, \xi\right\rangle+\left\|\mathscr{L}_{W} \xi\right\|^{2}
\end{gathered}
$$

Proof. Fix $p \in M_{0}$ and choose orthonormal vectors $e_{1}, \ldots, e_{n} \in \mathbf{T}_{p}\left(N_{p}\right)$ such that $\xi(p)=\mathbf{v}(p) \xi_{0}, \quad \xi_{0}=e_{1} \wedge \cdots \wedge e_{n}$. It is shown on p. 434 of [LS] that $\frac{1}{2}\left(L_{W} \sigma\right)\left(\xi_{0}, \xi_{0}\right)=\left\langle A^{W} \xi_{0}, \xi_{0}\right\rangle$, where $A^{W}: \wedge_{*} \mathbf{T}_{p}(M) \rightarrow \wedge_{*} T_{p}(M)$ is defined by $A^{W}(v)=\nabla_{v} W, v \in \mathbf{T}_{p}(M)$, and then extended to $\Lambda_{*} \mathbf{T}_{p}(M)$ as a derivation. Thus $A^{W} \xi_{0}=\sum_{i=1}^{n} e_{1} \wedge \cdots \wedge \nabla_{e_{i}} W \wedge \cdots \wedge e_{n}$, and

$$
\left\langle A^{W} \xi_{0}, \xi_{0}\right\rangle=\sum_{i=1}^{n}\left\langle\nabla_{e_{i}} W, e_{i}\right\rangle=-H^{W}(p) \text {. }
$$


To verify the second part of (i) we first assume $W$ to be invariant. Then the flow of $W$ preserves the fibres of $F$ and so referring to [L, p. 7] we have

$$
(W \mathbf{v})(p)=-\int_{N_{p}} H^{W} d \mathscr{H}^{n}=-H^{W}(p) \mathbf{v}(p) .
$$

Finally, $W \mathbf{v}=-H^{W} \mathbf{v}$ for arbitrary $W$ follows from the linearity in $W$ of both $W \mathbf{v}$ and $H^{W}$.

To verify (ii) first observe that since $\mathscr{L}_{W}$ commutes with contractions,

$$
\left(\mathscr{L}_{W} \sigma\right)\left(\eta_{1}, \eta_{2}\right)=W\left\langle\eta_{1}, \eta_{2}\right\rangle-\left\langle\mathscr{L}_{W} \eta_{1}, \eta_{2}\right\rangle-\left\langle\eta_{1}, \mathscr{L}_{W} \eta_{2}\right\rangle
$$

whenever $\eta_{1}, \eta_{2}$ are smooth $n$-vector fields on a neighborhood of $p$. Applying this with $\eta_{1}=\xi=\eta_{2}$ we obtain $\left(\mathscr{L}_{W} \sigma\right)(\xi, \xi)=W\left(\mathbf{v}^{2}\right)-2\left\langle\mathscr{L}_{W} \xi, \xi\right\rangle$ and infer using (i) that

$$
\left\langle\mathscr{L}_{W} \xi, \xi\right\rangle=0
$$

Applying (*) with $\eta_{1}=\xi, \eta_{2}=\mathscr{L}_{W} \xi$ we obtain $\left(\mathscr{L}_{W} \sigma\right)\left(\xi, \mathscr{L}_{W} \xi\right)=-\left\|\mathscr{L}_{W} \xi\right\|^{2}-$ $\left\langle\mathscr{L}_{W}^{2} \xi, \xi\right\rangle$. Finally, using again the fact that $\mathscr{L}_{W}$ commutes with contractions, we conclude that

$$
\begin{aligned}
\frac{1}{2}\left(\mathscr{L}_{W}^{2} \boldsymbol{\sigma}\right)(\xi, \xi) & =\frac{1}{2}\left[W\left[\left(\mathscr{L}_{W} \boldsymbol{\sigma}\right)(\xi, \xi)\right]-2\left(\mathscr{L}_{W} \boldsymbol{\sigma}\right)\left(\mathscr{L}_{W} \xi, \xi\right)\right] \\
& =W(\mathbf{v} W \mathbf{v})+\left\|\mathscr{L}_{W} \xi\right\|^{2}+\left\langle\mathscr{L}_{W}^{2} \xi, \xi\right\rangle .
\end{aligned}
$$

Fix $p \in M$ and choose a smooth $n$-vector field $\xi$ on an open subset $M_{0}$ as in the Lemma and such that in addition $\mathscr{H}^{n}\left(N_{p} \sim M_{0}\right)=0$.

Theorem. Let $W \in \mathscr{X}(M)$ be horizontal. Then

$$
\boldsymbol{\delta}_{p}^{(1)}(W)=\mathbf{v}(p)^{-1} \int_{N_{p}} W \mathbf{v} d \mathscr{H}^{n}
$$

$$
\boldsymbol{\delta}_{p}^{(2)}(W)=\int_{N_{p}} \mathbf{v}(p)^{-1} W^{2} \mathbf{v}+\left\langle\mathscr{L}_{W} \xi_{0}, \xi_{0}\right\rangle+\left\|\mathscr{L}_{W} \xi_{0}\right\|^{2} d \mathscr{H}^{n}
$$

where $\xi_{0}=\mathbf{v}(p)^{-1} \xi$.

Proof. Denote by $\phi_{t}$ the flow associated to $W$. $\left\|\phi_{t^{*}} \xi\right\|^{2}=\left(\phi_{t}^{*} \sigma\right)(\xi$, $\xi)$ hence

$$
\left.\frac{d}{d t}\left\|\phi_{t^{*}} \xi\right\|\right|_{t=0}=\mathbf{v}^{-1}\left(\mathscr{L}_{W} \boldsymbol{\sigma}\right)(\xi, \xi)=W \mathbf{v}
$$

and so the Lemma implies

$$
\left.\frac{d}{d t} \mathscr{H}^{n}\left(\phi_{t} N_{p}\right)\right|_{t=0}=\left.\frac{d}{d t} \int_{N_{p}}\left\|\phi_{t^{*}} \xi_{0}\right\| d \mathscr{H}^{n}\right|_{t=0}=\mathbf{v}(p)^{-1} \int_{N_{p}} W \mathbf{v} d \mathscr{H}^{n}
$$

Similarly,

$$
\left.\frac{d^{2}}{d t^{2}}\left\|\phi_{t^{*}} \xi\right\|\right|_{t=0}=(2 \mathbf{v})^{-1}\left(\mathscr{L}_{W}^{2} \sigma\right)(\xi, \xi)-\mathbf{v}^{-3}\left[\frac{1}{2}\left(\mathscr{L}_{W} \boldsymbol{\sigma}\right)(\xi, \xi)\right]^{2}
$$

3.2. Remarks. Inasmuch as the right side of (i) is linear in $W$, it follows from the Theorem that $N_{p}$ is minimal if and only if $W \mathbf{v}=0$ for each invariant horizontal vector field $W$. This is clearly equivalent to the statement that $N_{p}$ is minimal if and 
only if the function $\mathbf{v}_{*}$ on $G \backslash M$ corresponding to $\mathbf{v}$ is critical at $F(p)$, the condition discovered for principal orbits of isometry groups by W. Y. Hsiang $[\mathbf{H}]$; also see $[\mathbf{L}$, p. 21].

On the other hand, second variation cannot be analyzed so simply because of the fact that if $W$ is invariant and horizontal, then $\mathscr{L}_{W} \xi=0$ and so the right side of (ii) reduces to $\int_{N_{p}} \mathbf{v}(p)^{-1} W^{2} \mathbf{v} d \mathscr{H}^{n}$. (Indeed, it was shown in the proof of Lemma 3.1 that $\left\langle\mathscr{L}_{W} \xi, \xi\right\rangle=0$. However, $\mathscr{L}_{W} \xi$ is tangent to the fibres of $F$ because in the present case the flow of $W$ preserves the fibres.) Consequently, it would seem that information concerning the second derivative of $\mathbf{v}_{*}$ is not sufficient to determine stability of a minimal $N_{p}$.

3.3. For the remainder of this section we fix a critical point $p_{0}$ of $\mathbf{v}$ and denote $N=N_{p_{0}}, \boldsymbol{\delta}^{(k)}=\boldsymbol{\delta}_{p_{0}}^{(k)}$. We also fix invariant orthonormal normal vector fields $W_{1}, \ldots, W_{m-n}$ on $N$. Fix $p \in N$ and extend each $W_{i}(p)$ to a horizontal vector field $\tilde{W}_{i}$ defined in a neighborhood of $p$. Denote by $W_{i k}(p)$ the projection of $\left[\tilde{W}_{i}, \tilde{W}_{k}\right](p)$ onto $N$. Lemma 2 of [ON] implies that $W_{i k}(p)$ depends only on $W_{i}(p)$ and $W_{k}(p)$. Thus since each $\tilde{W}_{i}$ can in particular be chosen to be invariant, $W_{i k}$ is clearly smooth.

It is also shown in [ON] that if $G \backslash M$ is provided with the metric such that $F_{*} \mid \mathbf{T}_{p}\left(N_{p}\right)^{\perp}$ is an isometry for $p \in M$, then

$$
\left\|W_{i k}\right\|^{2}=\frac{4}{3}\left[K_{*}\left(F_{*} W_{i}, F_{*} W_{k}\right)-K\left(W_{i}, W_{k}\right)\right],
$$

where $K$ and $K_{*}$ are sectional curvatures in $M$ and $G \backslash M$, respectively.

For $i, k=1, \ldots, m-n$ denote

$$
\nu_{i}=\operatorname{Card}\left\{k: W_{i k} \neq 0\right\}, \quad \bar{W}_{i k}=\left(\nu_{i} \nu_{k}\right)^{1 / 2} W_{i k} .
$$

Assume $\nu_{i} \neq 0$ for $i \leqslant i_{1}$ and $\nu_{i}=0$ for $i>i_{1}$. Define $b_{i k}=1$ if $W_{i k} \neq 0$ and $b_{i k}=0$ otherwise.

Let $W=\sum_{i=1}^{m-n} a^{i} W_{i}$ be a smooth normal vector field on $N$. For $i, k=1, \ldots, i_{1}$ denote $\bar{a}^{i}=\nu_{i}^{-1 / 2} a^{i}, r^{i k}=\left[\left(\bar{a}^{i}\right)^{2}+\left(\bar{a}^{k}\right)^{2}\right]^{1 / 2}, A_{i k}=\left\{p: r^{i k}(p) \neq 0\right\}$, and define $\theta^{i k}: A_{i k} \rightarrow \mathbf{S}^{1}$ so that for $p \in A_{i k},\left(r^{i k}(p), \theta^{i k}(p)\right)$ are the polar coordinates of the orthogonal projection of $\bar{W}(p)=\sum_{i=1}^{m-n} \bar{a}^{i}(p) W_{i}(p)$ into the span of $\left\{W_{i}(p), W_{k}(p)\right\}$. Denote $d \theta^{i k}=\theta^{i k^{*}} \Omega$, where $\Omega$ is the positively oriented unit 1 -form on $\mathbf{S}^{1}$. We have

$$
d \theta^{i k}=\left(\nu_{i} \nu_{k}\right)^{-1 / 2}\left(r^{i k}\right)^{-2}\left(a^{i} d a^{k}-a^{k} d a^{i}\right) .
$$

Corollary.

$$
\begin{aligned}
\boldsymbol{\delta}^{(2)}(W)= & \int_{N} \mathbf{v}\left(p_{0}\right)^{-1} H_{\mathbf{v}}(W, W)+\sum_{i=1}^{m-n}\left\|d a^{i}\right\|^{2}-\sum_{i<k}\left\langle W_{i k}, a^{i} d a^{k}-a^{k} d a^{i}\right\rangle d \mathscr{H}^{n} \\
= & \int_{N} \mathbf{v}\left(p_{0}\right)^{-1} H_{\mathbf{v}}(W, W)+\sum_{i=i_{1}+1}^{m-n}\left\|d a^{i}\right\|^{2} d \mathscr{H}^{n} \\
& +\sum_{i<k}^{i_{1}} b_{i k} \int_{A_{i k}}\left\|d r^{i k}\right\|^{2}+\left(r^{i k}\right)^{2}\left(\left\|d \theta^{i k}\right\|^{2}-\left\langle\bar{W}_{i k}, d \theta^{i k}\right\rangle\right) d \mathscr{H}^{n}
\end{aligned}
$$


Proof. Extend $W_{1}, \ldots, W_{m-n}$ to smooth invariant orthonormal horizontal vector fields in a neighborhood of $N$, extend $W$ smoothly, and define $\xi, \xi_{0}$ as in 3.1 with $p_{0} \in M_{0}$. Let $X_{1}, \ldots, X_{m} \in \mathscr{X}(N)$ be orthonormal vector fields in a neighborhood of $p_{0}$ with $\xi_{0}=X_{1} \wedge \cdots \wedge X_{n}$. Denote the flow of $W_{i}$ by $\phi_{t}^{i}$. Denoting $M^{0}=N$ and inductively defining

$$
M^{i}=\bigcup_{t} \phi_{t}^{i}\left(M^{i-1}\right), \quad i=1, \ldots, m-n,
$$

we observe that there is a connected neighborhood $U$ of $p_{0}$ in which each $M^{i}$ is a manifold of dimension $n+i$, with $M^{m-n} \cap U=U$, and in which each $W_{i}$ has connected integral curves. For each $j$ we use the flows $\phi_{t}^{i}$ in succession to extend $X_{j}$ to $M^{i} \cap U$. It is clear that

$$
\left[W_{k}, X_{j}\right]=0=\left[W_{i},\left[W_{k}, X_{j}\right]\right] \text { on } N
$$

for $i, k=1, \ldots, m-n, i<k$, and $j=1, \ldots, n$. We also note that inasmuch as $\xi_{0}$ is invariant under each of the flows $\phi_{t}^{i}$ as was observed in 3.2 and $X_{1} \wedge \cdots \wedge X_{n}=\xi_{0}$ on $N$, we can conclude that $X_{1} \wedge \cdots \wedge X_{n}=\xi_{0}$ on $U$.

Expanding $\mathscr{L}_{W} \xi_{0}$ we obtain

$$
\begin{aligned}
\mathscr{L}_{W} \xi_{0}= & \sum_{i, j} a^{i} X_{i} \wedge \cdots \wedge\left[W_{i}, X_{j}\right]_{(j)} \wedge \cdots \wedge X_{n} \\
& -\left\langle X_{j}, d a^{i}\right\rangle X_{1} \wedge \cdots \wedge W_{i(j)} \wedge \cdots \wedge X_{n},
\end{aligned}
$$

where $W_{i(j)}$ indicates that $W_{i}$ occurs as the $j$ th factor, etc. In particular, on $N(*)$ implies $\left\|\mathscr{L}_{W} \xi_{0}\right\|^{2}=\sum_{i=1}^{m-n}\left\|d a^{i}\right\|^{2}$. Differentiating again, restricting to $N$ and using (*) and the Jacobi identity one obtains

$$
\begin{aligned}
&\left\langle\mathscr{L}_{W}^{2} \xi_{0}, \xi_{0}\right\rangle= \sum_{i, j} a^{i}\left\langle\mathscr{L}_{W}\left(X_{1} \wedge \cdots \wedge\left[W_{i}, X_{j}\right]_{(j)} \wedge \cdots \wedge X_{n}\right), \xi_{0}\right\rangle \\
&-\left\langle X_{j}, d a^{i}\right\rangle\left\langle\mathscr{L}_{W}\left(X_{1} \wedge \cdots \wedge W_{i(j)} \wedge \cdots \wedge X_{n}\right), \xi_{0}\right\rangle \\
&= \sum_{i, j, k} a^{i} a^{k}\left\langle\left[W_{k},\left[W_{i}, X_{j}\right]\right], X_{j}\right\rangle-a^{k}\left\langle X_{j}, d a^{i}\right\rangle\left\langle W_{k i}, X_{j}\right\rangle \\
&= \sum_{i<k}\left(\sum_{j} a^{i} a^{k}\left\langle-\left[W_{i},\left[X_{j}, W_{k}\right]\right]-\left[X_{j},\left[W_{k}, W_{i}\right]\right], X_{j}\right\rangle\right. \\
&\left.-\left\langle W_{i k}, a^{i} d a^{k}-a^{k} d a^{i}\right\rangle\right) \\
&= \sum_{i<k} a^{i} a^{k} \operatorname{div}_{N} W_{i k}-\left\langle W_{i k}, a^{i} d a^{k}-a^{k} d a^{i}\right\rangle
\end{aligned}
$$

because $\left\|X_{j}\right\|=1$ on $N$. Finally, $\operatorname{div}_{N} W_{i k}=0$ because $W_{i k}$ is invariant hence $\operatorname{div}_{N} W_{i k}$ is constant. The first formula is now clear.

Next we observe that

$$
\sum_{i=1}^{i_{1}}\left\|d a^{i}\right\|^{2}=\sum_{i<k}^{i_{1}} b_{i k}\left(\left\|d \bar{a}^{i}\right\|^{2}+\left\|d \bar{a}^{k}\right\|^{2}\right) .
$$


Fixing $i<k$ with $b_{i k}=1$ we see that on $A_{i k},\left\|d \bar{a}^{i}\right\|^{2}+\left\|d \bar{a}^{k}\right\|^{2}=\left\|d r^{i k}\right\|^{2}+$ $\left(r^{i k}\right)^{2}\left\|d \theta^{i k}\right\|^{2}$. On the other hand, $d \bar{a}^{i}$ and $d \bar{a}^{k}$ vanish at almost all points of $N \sim A_{i k}$ because $N \sim A_{i k}$ has positive Lebesgue density at almost all points of $N \sim A_{i k}$.

3.4. REMARK. The second variation formulas in 3.3 are valid in the more general situation that, instead of $N$ being a principal orbit of $G$, there exists a riemannian submersion $F: M \rightarrow P$ such that for each horizontal lift $W$ the restriction of $H^{W}$ to $N_{p}=F^{-1}\{F(p)\}$ is constant for $p \in M$. Then $N_{p}$ is minimal if and only if $\mathbf{v}$ is critical at $p$, and if $N_{p}$ is minimal the formulas in 3.3 hold.

\section{Stability.}

4.1. For this discussion we need merely assume the existence of a riemannian submersion $F: M \rightarrow P$ such that $N_{p}=F^{-1}\{F(p)\}, p \in M$. Assume there exists a smooth $n$-vector field $\xi$ on $M$ such that $\xi(p)$ is tangent to $N_{p}$ and $\|\xi(p)\|=\mathbf{v}(p)$ for $p \in M$. Denote by $\Omega$ the smooth $n$-form on $M$ dual to $\xi$. Orient each $N_{p}$ so that $\xi \mid N_{p}$ is positively oriented.

LEMMA. The horizontal distribution of $F$ is involutive if and only if $d \Omega=0$.

Proof. Let $W \in \mathscr{X}(M)$ be horizontal. Inasmuch as $\mathscr{L}_{W}$ commutes with contractions,

$0=W\langle\xi, \Omega\rangle=\left\langle\mathscr{L}_{W} \xi, \Omega\right\rangle+\left\langle\xi, \mathscr{L}_{W} \Omega\right\rangle=\left\langle\xi, d \iota_{W} \Omega\right\rangle+\left\langle\xi,_{W} d \Omega\right\rangle=\langle W \wedge \xi, d \Omega\rangle$

because $\iota_{W} \Omega=0$ and $(* *)$ in the proof of Lemma 3.1 implies that $\left\langle\mathscr{L}_{W} \xi, \Omega\right\rangle=0$. (Here $\iota_{W}$ is interior multiplication by $W[\mathbf{K N}]$.)

Referring to the formula for evaluation of $d \Omega$ on p. 36 of [ $\mathbf{K N}$, vol. I] we use this to conclude that if $X_{0}, X_{1}, \ldots, X_{n}$ are smooth vector fields in an open subset of $M$ which are either horizontal or vertical, then $d \Omega\left(X_{0}, \ldots, X_{n}\right)=0$ unless exactly two of these vectors, say $X_{0}$ and $X_{1}$, are horizontal. In this case

$$
d \Omega\left(X_{0}, \ldots, X_{n}\right)=-(n+1)^{-1} \Omega\left(\left[X_{0}, X_{1}\right], X_{2}, \ldots, X_{n}\right),
$$

and it is clear that the expression on the right vanishes for all such choices of $X_{0}, \ldots, X_{n}$ if and only if $\left[X_{0}, X_{1}\right]$ is horizontal whenever $X_{0}$ and $X_{1}$ are horizontal.

THEOREM. Assume the horizontal distribution of $F$ to be involutive. Then $N_{p}$ minimizes area among all rectifiable currents of dimension $n$ in $M$ which are homologous to $N_{p}$ if and only if $\mathbf{v}$ has an absolute minimum (not necessarily strict) at $p$.

Proof. Inasmuch as any two fibres of $F$ are homologous, necessity is clear. Thus assume $\mathbf{v}$ to have an absolute minimum at $p$. Since $d \Omega=0$ the following standard argument using the form $\Omega_{0}=\mathrm{v}(p) \Omega$ can be used to show $N_{p}$ area minimizing: Let $T$ be a rectifiable $n$-current which is homologous to $N_{p}$, which means that $N_{p}-T=$ $\partial S$ for some rectifiable $(n+1)$-current $S$. (See [F] for definitions of the terminology.) Then since $\left\|\Omega_{0}\right\|=\mathbf{v}(p) / \mathbf{v} \leqslant 1$, we have

$$
\mathbf{M}\left(N_{p}\right)=\mathscr{H}^{n}\left(N_{p}\right)=\int_{N_{p}} \Omega_{0}=\int_{\partial S} \Omega_{0}+\int_{T} \Omega_{0} \leqslant \mathbf{M}(T)\left\|\Omega_{0}\right\| \leqslant \mathbf{M}(T) .
$$


4.2. It is well known that currents with the property described in Theorem 4.1 are minimal and stable [F, p. 525]. The next result is the infinitesimal analogue of Theorem 4.1.

We will say that the distribution of normal planes to the orbits is involutive along $N_{p}$ if for orthonormal vector fields $W_{1}, \ldots, W_{m-n}$ defined on $N_{p}$ the vector fields $W_{i k}$ defined in $3.3, i, k=1, \ldots, m-n$, all vanish on $N_{p}$.

THEOREM. (i) $N_{p}$ is minimal if and only if $p$ is a critical point of $\mathbf{v}$.

(ii) Assume $p$ to be a critical point of $\mathbf{v}$. If $N_{p}$ is stable, then the hessian $H_{\mathbf{v}}(p)$ of $\mathbf{v}$ at $p$ is nonnegative. Conversely, if the distribution of normal planes to the orbits is involutive along $N_{p}$ and $H_{\mathrm{v}}(p) \geqslant 0$, then $N_{p}$ is stable.

Proof. (i) is discussed in 3.2; thus assume $p$ to be a critical point of $\mathbf{v}$.

Assume there exists $w \in \mathbf{T}_{p}\left(N_{p}\right)^{\perp}$ with $H_{\mathbf{v}}(p)(w, w)<0$. Let $W \in \mathscr{X}(M)$ be an invariant horizontal vector field with $W(p)=w$. It is clear that $H_{\mathrm{v}}(W, W)$ is constant on $N_{p}$, hence we infer using Corollary 3.3 that $\delta_{p}^{(2)}(W)=H_{\mathrm{v}}(W, W)<0$.

Conversely, if $H_{\mathbf{v}}(p) \geqslant 0$ and the distribution of normal planes is involutive along $N_{p}$, then for $W$ horizontal we infer that $\delta_{p}^{(2)}(W) \geqslant \mathbf{v}(p)^{-1} \int_{N_{p}} H_{\mathrm{v}}(W, W) d \mathscr{H}^{n} \geqslant 0$.

4.3. For the remainder of $\S 4$ we fix a critical point $p_{0}$ of $\mathbf{v}$ and readopt the notation of 3.3. Our goal is to strengthen the stability criterion of 4.2 by showing that $N$ will be stable provided $H_{\mathbf{v}} \geqslant 0$ and the numbers $\left\|\bar{W}_{i k}\right\|=\left(\nu_{i} \nu_{k}\right)^{1 / 2}\left\|W_{i k}\right\|$ are not too large. Denote the largest of these numbers by $\mu$, and denote $\lambda=$ $\inf \mathbf{v}(p)^{-1} H_{\mathbf{v}}(p)(w, w)$, where the infimum is taken over $\mathbf{T}_{p}(N)^{\perp} \cap\{w:\|w\|=1\}$ (and does not depend on $p \in N$ ). Note that $\mu=0$ if and only if the distribution of normal planes to the orbits is involutive along $N$.

THEOREM. If $\lambda \geqslant \mu^{2} / 4$, then $N$ is stable.

Proof. For $W=\sum_{i=1}^{m-n} a^{i} W_{i}$ we have by Corollary 3.3

$$
\delta^{(2)}(W) \geqslant \sum_{i<k} b_{i k} \int_{A_{i k}}\left(r^{i k}\right)^{2}\left(\left\|d \theta^{i k}\right\|^{2}-\left\|\bar{W}_{i k}\right\|\left\|d \theta^{i k}\right\|+\lambda\right) d \mathscr{H}^{n} \geqslant 0
$$

because the polynomial $x^{2}-\mu x+\lambda \geqslant 0$ for $\mu^{2} \leqslant 4 \lambda$.

4.4. Lemma. Denote by $C_{l}$ the circle of length $l$ with unit positively oriented tangent vector field $X$. For $a^{1}, a^{2} \in C^{\infty}\left(C_{l}\right)$ and $c \in \mathbf{R}$ denote

$$
I_{c}\left(a^{1}, a^{2}\right)=\int_{C_{l}}\left\|d a^{1}\right\|^{2}+\left\|d a^{2}\right\|^{2}-c\left\langle X, a^{1} d a^{2}-a^{2} d a^{1}\right\rangle d \mathscr{H}^{1} .
$$

(i) If $|c| l \leqslant 2 \pi$, then $I_{c} \geqslant 0$.

(ii) If $|c| l>2 \pi$, then inf $I_{c}=-\infty$.

Proof. We can assume $l=2 \pi$ and, because $I_{c}\left(a^{1}, a^{2}\right)=I_{-c}\left(a^{2}, a^{1}\right)$, that $c>0$.

Let $\mathbf{U}(2)$ have the bi-invariant metric induced from the standard hermitian inner product of $g \ell(2, \mathbf{C})$ by the inclusion of $\mathbf{U}(2)$ in $g \ell(2, \mathbf{C})$. Embed $C_{2 \pi}=\mathbf{U}(1)$ in $\mathbf{U}(2)$ as matrices of the form

$$
\left[\begin{array}{cc}
e^{i \theta} & 0 \\
0 & 1
\end{array}\right]
$$


$\mathbf{U}(1)$ is an orbit of the action of $\mathbf{U}(1)$ on $\mathbf{U}(2)$ by left multiplication.

$$
W_{1}=2^{-1 / 2}\left[\begin{array}{cc}
0 & -1 \\
1 & 0
\end{array}\right], \quad W_{2}=2^{-1 / 2}\left[\begin{array}{cc}
0 & i \\
i & 0
\end{array}\right]
$$

are orthonormal vectors normal to $\mathbf{U}(1)$ at $I$; extend $W_{1}, W_{2}$ to normal vector fields on $\mathbf{U}(1)$ by left translation. In view of the discussion in 5.1 we see that $W_{12}(I)$ is the projection on $\mathbf{U}(1)$ of

$$
-\left[W_{1}(I), W_{2}(I)\right]=\left[\begin{array}{cc}
i & 0 \\
0 & -i
\end{array}\right],
$$

which is $X(I)$. Thus $W_{12}=X$.

We next show that $\mathbf{U}(1)$ is of least length in its homology class. Indeed, let $C$ be a minimizing geodesic through $I$ which is homologous to $\mathbf{U}(1)$. Then $C$ is a oneparameter subgroup. Since the rank of $\mathbf{U}(2)$ is $2, C$ is contained in a maximal torus $\mathbf{T}^{2}$, which is conjugate to $\mathbf{S}^{1} \times \mathbf{S}^{1}$ with the product metric. But $\mathscr{H}^{1}(C) \leqslant \mathscr{H}^{1}(\mathbf{U}(1))$ $=2 \pi$ and so $C$ is conjugate to $\mathbf{U}(1)$.

We infer using Corollary 3.3 that the second variation of length of $\mathbf{U}(1)$ with respect to $W=a^{1} W_{1}+a^{2} W_{2}$ is equal to $I_{1}\left(a^{1}, a^{2}\right)$, hence conclude using [F, 5.1.7] that $I_{1}\left(a^{1}, a^{2}\right) \geqslant 0$. Finally, we observe that if $0<c \leqslant 1$, then $c^{-1} I_{c}\left(a^{1}, a^{2}\right) \geqslant$ $I_{1}\left(a^{1}, a^{2}\right) \geqslant 0$.

Defining $r=r^{12}=\left[\left(a^{1}\right)^{2}+\left(a^{2}\right)^{2}\right]^{1 / 2}$ and, assuming $r>0$ and $\theta=\theta^{12}$ as in 3.3 we have $a^{1}=r \cos \theta, a^{2}=r \sin \theta$,

$$
I_{c}\left(a^{1}, a^{2}\right)=\int_{C_{2 \pi}}\|d r\|^{2}+r^{2}\left(\|d \theta\|^{2}-c\langle X, d \theta\rangle\right) d \mathscr{H}^{1} .
$$

In case $\theta$ is the identity and $r$ is constant this reduces to $I_{c}\left(a^{1}, a^{2}\right)=2 \pi r^{2}(1-c)$. (ii) is now clear for $c>1$.

4.5. Assume there exist invariant orthonormal vector fields $X_{1}, \ldots, X_{n}$ on $N$ such that the trajectories of each $X_{j}$ are compact; these have constant length $L_{j}$ since $X_{j}$ is invariant. Recall the definitions of $W_{i k}$ and $\nu_{i}$ in 3.3 .

THEOREM. If $H_{\mathrm{v}} \geqslant 0$ and $\left(\nu_{i} \nu_{k}\right)^{1 / 2}\left|\left\langle W_{i k}, X_{j}\right\rangle\right| \leqslant 2 \pi / L_{j}$ for every $i, k$, $j$, then $N$ is stable.

Proof. Let $W=\sum_{i=1}^{m-n} a^{i} W_{i}$ be smooth on $N$. Corollary 3.3 implies that

$$
\delta^{(2)}(W) \geqslant \sum_{i<k} \int_{N} b_{i k}\left(\left\|d \bar{a}^{i}\right\|^{2}+\left\|d \bar{a}^{k}\right\|^{2}\right)-\left\langle\bar{W}_{i k}, \bar{a}^{i} d \bar{a}^{k}-\bar{a}^{k} d \bar{a}^{i}\right\rangle d \mathscr{H}^{n} .
$$

Fix $i<k$ with $b_{i k}=1$. Then the corresponding term in the above sum is equal to

(*) $\sum_{j=1}^{n} \int_{N}\left\langle X_{j}, d \bar{a}^{i}\right\rangle^{2}+\left\langle X_{j}, d \bar{a}^{k}\right\rangle^{2}-\left\langle\bar{W}_{i k}, X_{j}\right\rangle\left\langle X_{j}, \bar{a}^{i} d \bar{a}^{k}-\bar{a}^{k} d \bar{a}^{i}\right\rangle d \mathscr{H}^{n}$.

Fixing $j$ we infer that, since the action of $G$ commutes with the flow of $X=X_{j}$, the set $P$ of orbits of $X$ has a smooth manifold structure and the map $\Phi$ which associates the trajectory of $p$ with each $p \in N$ is a submersion. Fix a riemannian metric on $P$. We next show that the jacobian $J \Phi$ of $\Phi$ is constant on $\Phi^{-1}\{q\}$ for each $q \in P$. Fix $v \in \Lambda_{n-1} \mathbf{T}_{q}(P)$ with $\|v\|=1$, and for $p \in \Phi^{-1}\{q\}$ let $v^{*}(p)$ be the 
horizontal lift of $v$ to $p$. Then $J \Phi(p)=\left\|v^{*}(p)\right\|^{-1}$. Denoting the flow of $X$ by $\phi_{t}$ we have $\phi_{t^{*}}\left(v^{*} \wedge X\right)=\left(\phi_{t^{*}} v^{*}\right) \wedge X=v^{*} \wedge X$ because $\Phi_{*} \phi_{t^{*}}\left(v^{*}\right)=v$ and $\phi_{t^{*}} X=X$. From this we infer that, since $\operatorname{div} X=0,\left\|v^{*} \wedge X\right\|$ is constant on $\Phi^{-1}\{q\}$. Consequently, $\left\|v^{*}\right\|=\left\|v^{*} \wedge X\right\|\|X\|^{-1}$ is also constant.

Denoting $(J \Phi)^{-1}(q)=J \Phi(p)^{-1}$ for $\Phi(p)=q$ we use the coarea formula $[\mathbf{F}$, 3.2.12] to write the term in $(*)$ corresponding to $j$ in the form $\int_{P} I(q)(J \Phi)^{-1}(q) d \mathscr{H}^{n-1} q$, where for $q \in P$

$$
I(q)=\int_{\Phi^{-1}\{q\}}\left\langle X, d \bar{a}^{i}\right\rangle^{2}+\left\langle X, d \bar{a}^{k}\right\rangle^{2}-c_{i k}\left\langle X, \bar{a}^{i} d \bar{a}^{k}-\bar{a}^{k} d \bar{a}^{i}\right\rangle d \mathscr{H}^{1},
$$

and $c_{i k}=\left\langle\bar{W}_{i k}, X\right\rangle$. Finally, $\left|c_{i k}\right| \leqslant 2 \pi / L_{j}$ by hypothesis, hence Lemma 4.4 implies $I(q) \geqslant 0$.

4.6. Assume the following:

(i) There exist mutually orthogonal $G$-invariant distributions $A_{1}, \ldots, A_{\mu}$ on $N$ such that for each $i=1, \ldots, i_{1}$ and each $k$ with $W_{i k} \neq 0$ (see 3.3) there exists $j(i, k)$ such that $W_{i k} \in A_{j(i, k)}$.

(ii) For each $i$ the function $j(i, \cdot)$ is one-to-one.

(iii) For each $j=1, \ldots, \mu$ there exists an invariant orthonormal basis $\left\{X_{j 1}, \ldots, X_{j \mu}\right\}$ of $A_{j}$ such that each vector field $X_{j l}$ has compact orbits. Denote by $L_{j l}$ the length of the orbits of $X_{j l}$.

THEOREM. If $H_{\mathrm{v}} \geqslant 0$ and $\left|\left\langle W_{i k}, X_{j(i, k), l}\right\rangle\right| \leqslant 2 \pi / L_{j(i, k), l}$ for every $i, k, l$, then $N$ is stable.

Proof. Let $W=\sum_{i=1}^{m-n} a^{i} W_{i}$ be smooth on $N$. Then by Corollary 3.3

$$
\begin{aligned}
\delta^{(2)}(W) \geqslant & \sum_{i<k} \sum_{l} \int_{N}\left\langle X_{j(i, k), l}, d a^{i}\right\rangle^{2}+\left\langle X_{j(i, k), l}, d a^{k}\right\rangle^{2} \\
& -c_{i k l}\left\langle X_{j(i, k), l}, a^{i} d a^{k}-a^{k} d a^{i}\right\rangle d \mathscr{H}^{n},
\end{aligned}
$$

where $c_{i k l}=\left\langle W_{i k}, X_{j(i, k), l}\right\rangle$, which is nonnegative by an argument analogous to the second part of the proof of 4.5 .

4.7. Lemma. Fix $i, k$ with $W_{i k} \neq 0$. There exists a torus $\mathbf{T}^{l}$ of dimension $l$ which acts smoothly and freely on the right on $N$, thereby inducing on $N$ the structure of a principal fibre bundle with projection $\Phi: N \rightarrow N / \mathbf{T}^{l}$. Furthermore,

(i) the action of $\mathbf{T}^{\prime}$ on $N$ commutes with the action of $G$, and

(ii) for each $p \in N$ the orbit $(p) \mathbf{T}^{l}$ is equal to the closure of the orbit of $W_{i k}$ through $p$.

Proof. Denote $H=G \cap\left\{g: g\left(p_{0}\right)=p_{0}\right\}$ and $\Pi: G \rightarrow N, \Pi(g)=g\left(p_{0}\right)$. Choose an $\operatorname{Ad}(H)$-invariant subspace $\mathbf{V}$ of $\mathbf{T}_{e}(G)$ of dimension $n$ such that $\mathbf{V} \cap$ $\mathbf{T}_{e}(H)=\{0\}$ and denote by $W^{*}$ the left invariant vector field on $G$ such that $\Pi_{*}\left(W^{*}\right)=W_{i k}, W^{*}(e) \in \mathbf{V}$. $G$-invariance of $W_{i k}$ implies that $\operatorname{Ad}(H)$ fixes $W^{*}$, hence that $H$ lies in the centralizer of the one-parameter subgroup $\exp t W^{*}$. The closure in $G$ of this one-parameter subgroup is thus a torus $\mathbf{T}_{W^{*}}^{\lambda}$ containing $H$ in its centralizer. The component of $\mathbf{T}_{W^{*}}^{\lambda} \cap H$ containing $e$ is a torus $\mathbf{T}_{W^{*}}^{\lambda_{1}}$ and so $\mathbf{T}_{W^{*}}^{\lambda}=\mathbf{T}_{W^{*}}^{l} \times \mathbf{T}_{W^{*}}^{\lambda_{1}}$ 
Next we observe that because $H$ lies in the centralizer of $\mathbf{T}_{W^{*}}^{l}$, the action of $\mathbf{T}_{W^{*}}^{l}$ on $G$ by right translation induces a right action on $N$ which is $\Pi$-equivariant and commutes with the action of $G$. The isotropy groups of this action of $\mathbf{T}_{W^{*}}^{l}$ are thus all equal to the finite group $\mathbf{T}_{W^{*}}^{l} \cap H$. Consequently, the action of $\mathbf{T}_{W^{*}}^{l}$ induces an action of $\mathbf{T}^{l}$ on $N$ which is without fixed points, hence induces a principal fibration $\Phi: N \rightarrow N / \mathbf{T}^{\prime}$.

Finally, since $\Pi($ closure $S)=$ closure $\Pi(S)$ for $S \subset G$, we conclude that $\Pi\left(\mathbf{T}_{W^{*}}^{l}\right)$ $=\left(p_{0}\right) \mathbf{T}^{\prime}$ is the closure of the orbit of $W_{i k}$ through $p_{0}$; inasmuch as $W_{i k}$ is $G$-invariant, the closure of the orbit through $g\left(p_{0}\right)$ is $g\left(p_{0}\right) \mathbf{T}^{l}$.

4.8. For $i<k$ such that $W_{i k} \neq 0$ denote the associated torus of 4.7 by $\mathbf{T}^{l_{i k}}$ and by $\left\{X_{i k, 1}, \ldots, X_{i k, l_{i k}}\right\}$ a basis of the integer lattice $\exp ^{-1}\{e\}$ of $\mathbf{T}^{l_{i k}}$. We also denote by $X_{i k, j}$ the fundamental vector field of $N$ corresponding to $X_{i k, j} . X_{i k, j}$ is $G$-invariant because the actions of $G$ and $\mathbf{T}^{l_{i k}}$ commute. Defining $\nu_{i}$ as in 3.3 we also denote by $\lambda_{i k}$ and $\mu_{i k}$ respectively the infimum and supremum of

$$
\left\{\|v\|: v=\sum_{j=1}^{l_{i k}} v^{j} X_{i k, j}, \sum_{j=1}^{l_{i k}}\left|v^{j}\right|^{2}=1\right\} .
$$

THEOREM. If $H_{\mathbf{v}} \geqslant 0$ and $\left(\nu_{i} \nu_{k}\right)^{1 / 2}\left\|W_{i k}\right\| \leqslant 2 \pi \lambda_{i k} / \mu_{i k}^{2}$ for every $i, k$, then $N$ is stable.

Proof. The proof is an extension of the proof of Theorem 4.5. Fix $i<k$ with $b_{i k}=1$ and write $\bar{W}_{i k}=\sum_{j=1}^{l_{i k}} \bar{w}_{i k}^{j} X_{i k, j}$. Denote by ||$_{e}$ the norm on the tangent spaces of the orbits of $\mathbf{T}^{l_{i k}}$ induced by the inner product which makes the vector fields $X_{i k, j}, j=1, \ldots, l_{i k}$, orthonormal. Then $\lambda_{i k}||_{e} \leqslant\|\| \leqslant \mu_{i k}||_{e}$ on these tangent spaces, hence $\left\|d \bar{a}^{i}\right\|^{2} \geqslant \mu_{i k}^{-2} \sum_{j=1}^{l_{i k}}\left\langle X_{i k, j}, d \bar{a}^{i}\right\rangle^{2}$. Thus

$$
\begin{aligned}
& \int_{N}\left\|d \bar{a}^{i}\right\|^{2}+\left\|d \bar{a}^{k}\right\|^{2}-\left\langle\bar{W}_{i k}, \bar{a}^{i} d \bar{a}^{k}-\bar{a}^{k} d \bar{a}^{i}\right\rangle d \mathscr{H}^{n} \\
& \geqslant \sum_{j=1}^{l_{i k}}\left(\left\|X_{i k, j}\right\| / \mu_{i k}\right)^{2} \int_{N}\left\|X_{i k, j}\right\|^{-2}\left(\left\langle X_{i k, j}, d \bar{a}^{i}\right\rangle^{2}+\left\langle X_{i k, j}, d \bar{a}^{k}\right\rangle^{2}\right) \\
&-\bar{w}_{i k}^{j}\left(\mu_{i k} /\left\|X_{i k, j}\right\|\right)^{2}\left\langle X_{i k, j}, \bar{a}^{i} d \bar{a}^{k}-\bar{a}^{k} d \bar{a}^{i}\right\rangle d \mathscr{H}^{n}
\end{aligned}
$$

each term of which is nonnegative by an argument analogous to one used in the proof of 4.5 because the length of the orbits of $X_{i k, j}$ is equal to $\left\|X_{i k, j}\right\|$ and $\left|\bar{w}_{i k}^{j}\right| \leqslant\left|\bar{W}_{i k}\right|_{e} \leqslant \lambda_{i k}^{-1}\left\|\bar{W}_{i k}\right\| \leqslant 2 \pi \mu_{i k}^{-2}$.

4.9. Write the metric tensor $\sigma$ of $M$ as $\sigma=\sigma_{h}+\sigma_{v}$, where $\sigma_{v}(\xi, \eta)=0$ if $\xi$ or $\eta$ is horizontal and $\sigma_{h}(\xi, \eta)=0$ if $\xi$ or $\eta$ is vertical. For $c>0$ denote $\sigma_{c}=\sigma_{h}+c \sigma_{v}$.

Theorem. Assume $H_{\mathrm{v}} \geqslant 0$. Then there exists $c_{s} \in(0, \infty]$ such that $N$ is stable for $c \leqslant c_{s}\left(c<c_{s}\right.$ if $\left.c_{s}=\infty\right)$ and $N$ is not stable for $c>c_{s}$. Furthermore, $c_{s}=\infty$ and if and only if the distribution of normal planes to the orbits is involutive along $N$ (see 4.2).

Proof. It is clear from Corollary 3.3 that the integrand of $\delta^{(2)}(W)$ is a continuous decreasing function of $c$. Thus the set of $c$ for which $N$ is stable is a nonempty interval. 
In case the distribution of normal planes to the orbits is involutive along $N$, stability for all $c$ is implied by 4.2 . Otherwise, if there exist orthonormal normal vector fields $W_{1}, W_{2}$ on $N$ such that the projection $W_{12}$ of $\left[W_{1}, W_{2}\right]$ on $N$ is not 0 , then we choose $\theta: N \rightarrow \mathbf{S}^{1}$ such that $\left\langle W_{12}, d \theta\right\rangle$ has positive values, choose $r \geqslant 0$ so that

$$
\int_{N} r^{2}\left\langle W_{12}, d \theta\right\rangle d \mathscr{H}^{n}>0
$$

and consider the variation $W=r\left(\cos \theta W_{1}+\sin \theta W_{2}\right)$.

5. Examples. We consider the stability of various subgroups of the unitary group $\mathbf{U}(n)$.

Let $\mathbf{U}(n)$ have the bi-invariant metric induced from the standard hermitian inner product of $g \ell(n, \mathbf{C})$ by the inclusion of $\mathrm{U}(n)$ in $g \ell(n, \mathbf{C})$. Denote by $E_{\alpha \beta}$ the $n \times n$ matrix with 1 in row $\alpha$, column $\beta$, and 0 elsewhere. Then the set $\mathscr{B}_{n}$ of matrices

$$
I_{\alpha \beta}=2^{-1 / 2}\left(E_{\beta \alpha}-E_{\alpha \beta}\right), \quad J_{\alpha \beta}=2^{-1 / 2} i\left(E_{\alpha \beta}+E_{\beta \alpha}\right), \quad D_{\gamma}=i E_{\gamma \gamma}
$$

with $1 \leqslant \alpha<\beta \leqslant n$ and $1 \leqslant \gamma \leqslant n$ forms an orthonormal basis of $\mathbf{T}_{I}(\mathbf{U}(n))$. We also denote by $I_{\alpha \beta}, J_{\alpha \beta}$ and $D_{\gamma}$ the extensions of these to left invariant vector fields on $\mathrm{U}(n)$. Finally, denote $D_{\alpha \beta}=2^{-1 / 2}\left(D_{\alpha}-D_{\beta}\right)$. One verifies that for $\alpha_{1} \neq \alpha_{2}$

$$
\begin{gathered}
{\left[I_{\alpha_{1} \beta}, I_{\alpha_{2} \beta}\right]=2^{-1 / 2} I_{\alpha_{1} \alpha_{2}}=\left[J_{\alpha_{1} \beta}, J_{\alpha_{2} \beta}\right],} \\
{\left[I_{\alpha_{1} \beta}, J_{\alpha_{2} \beta}\right]=-2^{-1 / 2} J_{\alpha_{1} \alpha_{2}}, \quad\left[I_{\alpha \beta}, J_{\alpha \beta}\right]=D_{\beta}-D_{\alpha},} \\
{\left[I_{\alpha \beta}, D_{\alpha}\right]=J_{\alpha \beta}=-\left[I_{\alpha \beta}, D_{\beta}\right], \quad\left[J_{\alpha \beta}, D_{\alpha}\right]=-I_{\alpha \beta}=-\left[J_{\alpha \beta}, D_{\beta}\right] ;}
\end{gathered}
$$

all other brackets of members of the above basis are zero.

We will apply our theory as follows: $M$ and $N$ will be subgroups of $\mathbf{U}(n)$ with $N$ acting on $M$ by left multiplication, hence $\mathbf{T}(N)^{\perp}$ will have an orthonormal basis $\left\{W_{1}, \ldots, W_{\nu}\right\}$ consisting of left invariant vector fields. However, each $W_{i}$ will not in general be horizontal off $N$ because the orbits of $N$ are permuted by right translation by elements of $M$. Recalling 3.3 we compute the $N$-invariant vector fields $W_{i k}$ as follows: Let $\tilde{W}_{i}$ be the right invariant vector field such that $\tilde{W}_{i}(I)=W_{i}(I)$; $\tilde{W}_{i}$ is clearly horizontal. Further, $\left[\tilde{W}_{i}, \tilde{W}_{k}\right](I)=-\left[W_{i}, W_{k}\right](I)$, hence $W_{i k}$ is the projection on $N$ of $-\left[W_{i}, W_{k}\right]$.

5.1. ThEOREM. Let $1 \leqslant k_{1}<k_{2}<\cdots<k_{\nu} \leqslant n$ be integers and embed

$$
N=\mathbf{U}\left(k_{1}\right) \times \mathbf{U}\left(k_{2}-k_{1}\right) \times \cdots \times \mathbf{U}\left(k_{\nu}-k_{\nu-1}\right)
$$

as a subgroup of $\mathrm{U}(n)$ is one of the standard ways. Then $N$ is stable. In particular, a maximal torus of $\mathbf{U}(n)$ is stable.

Proof. We assume $\mathbf{T}(N)$ to be spanned by the set $\mathscr{B}(N)$ of $I_{\alpha \beta}, J_{\alpha \beta}, D_{\gamma}$ with $k_{\mu-1}<\alpha<\beta \leqslant k_{\mu}, \mu=1, \ldots, \nu\left(k_{0}=0\right), 1 \leqslant \gamma \leqslant k_{\nu} . \mathbf{T}(N)^{\perp}$ is thus spanned by $\mathscr{B}(N)^{\perp}=\mathscr{B}_{N} \sim \mathscr{B}(N)$. 
Denoting by $P$ the orthogonal projection onto $\mathbf{T}(N)$ we have for members of $\mathscr{B}(N)^{\perp}$

$$
\begin{gathered}
P\left[I_{\alpha_{1} \beta}, J_{\alpha_{2} \beta}\right]=2^{-1 / 2} I_{\alpha_{1} \alpha_{2}}=P\left[J_{\alpha_{1} \beta}, J_{\alpha_{2} \beta}\right], \\
P\left[I_{\alpha_{1} \beta}, J_{\alpha_{2} \beta}\right]=2^{-1 / 2} J_{\alpha_{1} \alpha_{2}}, \quad k_{\mu-1}<\alpha_{1}<\alpha_{2} \leqslant k_{\mu} ; \\
P\left[I_{\alpha \beta}, J_{\alpha \beta}\right]=\left\{\begin{array}{lc}
-D_{\alpha}, & 1 \leqslant \alpha \leqslant k_{\nu}<\beta \leqslant n, \\
D_{\beta}-D_{\alpha}, & 1 \leqslant \alpha<\beta \leqslant k_{\nu} ;
\end{array}\right.
\end{gathered}
$$

all other members of $\left[\mathscr{B}(N)^{\perp}, \mathscr{B}(N)^{\perp}\right]$ project to zero. Further, the orbits of $I_{\alpha \beta}$ and $J_{\alpha \beta}$ have length $2 \pi \sqrt{2}$ while those of $D_{\gamma}$ have length $2 \pi$. We can therefore apply Theorem 4.6 with $A_{1}$ equal to the span of $\left\{D_{1}, \ldots, D_{k_{v}}\right\}$ and $A_{j}, j>1$, equal to the span of one of the $I_{\alpha \beta}$ or $J_{\alpha \beta} \in \mathscr{B}(N)$.

REMARK. It is asserted in [T] that $\mathbf{U}(k)$ does not homologically minimize area in $\mathbf{U}(n)$, but I am unable to follow the proof. The above result is consistent with such a conjecture; however, it follows as in the proof of Lemma 4.4 that $\mathbf{U}(1)$ minimizes length in its homology class in $\mathrm{U}(n)$ for each $n$.

5.2. THEOREM. Let $\varepsilon_{1}, \ldots, \varepsilon_{\nu}$ be positive integers with $\sum_{\mu=1}^{\nu} 2^{\varepsilon_{\mu}} \leqslant n$ and embed

$$
N=\mathbf{S U}\left(2^{\varepsilon_{1}}\right) \times \cdots \times \mathbf{S U}\left(2^{\varepsilon_{\nu}}\right)
$$

as a subgroup of $\mathbf{U}(n)$ in one of the standard ways. Then $N$ is stable.

Proof. Denote $k_{\mu}=\sum_{\lambda=1}^{\mu} 2^{\varepsilon_{\lambda}}, \mu=1, \ldots, \nu, k_{0}=0$. We can assume $\mathbf{T}(N)$ to be spanned by the set of $I_{\alpha \beta}, J_{\alpha \beta}, D_{\alpha, \alpha+1}$ with $k_{\mu-1}<\alpha<\beta \leqslant k_{\mu}, \mu=1, \ldots, \nu$. The remaining $I_{\alpha \beta}, J_{\alpha \beta}$, together with an orthonormal basis of the orthogonal complement in the span of $\left\{D_{1}, \ldots, D_{n}\right\}$ of the span of $\left\{D_{\gamma, \gamma+1} \in \mathbf{T}(N)\right\}$, form an orthonormal basis $\mathscr{B}(N)^{\perp}$ of $\mathbf{T}(N)^{\perp}$.

Denoting by $P$ the orthogonal projection onto $\mathrm{T}(N)$ we have for members of $\mathscr{B}(N)^{\perp}$

$$
\begin{gathered}
P\left[I_{\alpha_{1} \beta}, I_{\alpha_{2} \beta}\right]=2^{-1 / 2} I_{\alpha_{1} \alpha_{2}}=P\left[J_{\alpha_{1} \beta}, J_{\alpha_{2} \beta}\right], \\
P\left[I_{\alpha_{1} \beta}, J_{\alpha_{2} \beta}\right]=2^{-1 / 2} J_{\alpha_{1} \alpha_{2}}, \quad k_{\mu-1}<\alpha_{1}<\alpha_{2} \leqslant k_{\mu} ; \\
P\left[I_{\alpha \beta}, J_{\alpha \beta}\right]=P\left(D_{\beta}-D_{\alpha}\right) ;
\end{gathered}
$$

all other members of $\left[\mathscr{B}(N)^{\perp}, \mathscr{B}(N)^{\perp}\right]$ project to zero.

We will apply Theorem 4.6 with $A_{1}$ equal to the span of $\left\{D_{\gamma, \gamma+1} \in \mathbf{T}(N)\right\}$ and $A_{j}, j>1$, equal to the span of one of the $I_{\alpha \beta}$ or $J_{\alpha \beta} \in \mathbf{T}(N)$. We also define an orthonormal basis $\left\{X_{\mu \lambda}: \mu=1, \ldots, \nu, 1 \leqslant \lambda<2^{\varepsilon_{\mu}}\right\}$ of $A_{1}$ by $X_{\mu \lambda}=D_{k_{\mu-1}+\lambda, k_{\mu-1}+\lambda+1}$ for $\lambda$ odd, and for $\lambda$ even

$$
X_{\mu \lambda}=2^{-\varepsilon_{\mu} / 2} \sum_{\gamma=1}^{2^{\varepsilon_{\mu}-1}} \varepsilon_{\mu / \gamma}\left(D_{k_{\mu-1}+2 \gamma-1}+D_{k_{\mu-1}+2 \gamma}\right), \quad l=\frac{\lambda}{2},
$$

where for each $\mu, \varepsilon_{\mu l \gamma}$ is the entry in row $l$, column $\gamma$ of the $\left(2^{\varepsilon_{\mu}-1}-1\right) \times 2^{\varepsilon_{\mu}-1}$

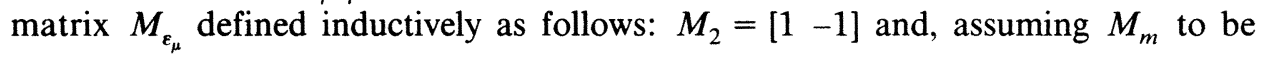


defined with $m \geqslant 2$,

$$
M_{m+1}=\left[\begin{array}{c|c}
M_{m} & M_{m} \\
M_{m} & -M_{m} \\
1 \cdots 1 & -1 \cdots-1
\end{array}\right]
$$

The orbits of $I_{\alpha \beta}, J_{\alpha \beta}$ and $X_{\mu \lambda}$ for $\lambda$ odd have length $2 \pi \sqrt{2}$; those of $X_{\mu \lambda}$ for $\lambda$ even have length $2 \pi 2^{\varepsilon_{\mu} / 2}$. Further, for $k_{\mu_{0}-1}<\alpha \leqslant k_{\mu_{0}}, k_{\mu_{1}-1}<\beta \leqslant k_{\mu_{1}}$ with $\mu_{0}<\mu_{1}$,

$$
\begin{aligned}
\left|\left\langle P\left(D_{\beta}-D_{\alpha}\right), X_{\mu \lambda}\right\rangle\right| & =0, \quad \mu \notin\left\{\mu_{0}, \mu_{1}\right\} \\
& =2^{-1 / 2} \text { or } 0, \quad \mu \in\left\{\mu_{0}, \mu_{1}\right\}, \lambda \text { odd } \\
& =2^{-\varepsilon_{\mu} / 2} \text { or } 0, \quad \mu \in\left\{\mu_{0}, \mu_{1}\right\}, \lambda \text { even. }
\end{aligned}
$$

In case $k_{\mu_{0}-1}<\alpha \leqslant k_{\mu_{0}}$ and $k_{\nu}<\beta$ the same is true with $\left\{\mu_{0}, \mu_{1}\right\}$ replaced by $\left\{\mu_{0}\right\}$. Finally, in case $k_{\nu}<\alpha<\beta \leqslant n, P\left(D_{\beta}-D_{\alpha}\right)=0$. Thus the requirements of Theorem 4.6 are satisfied; we conclude that $N$ is stable.

REMARKs. Our theory is inconclusive when applied to subgroups of $\mathbf{S U}(n)$ other than those listed in the Theorem. For example, in case $N=\mathbf{S U}(3)$ it is not possible to find an orthonormal basis $\left\{X_{1}, X_{2}\right\}$ of the span $A_{1}$ of $\left\{D_{12}, D_{23}\right\}$ such that the projections of $D_{\beta}-D_{\alpha}=\left[I_{\alpha \beta}, J_{\alpha \beta}\right], \alpha \leqslant 3, \beta>3$, each have components in the $X_{\lambda}$ directions, $\lambda=1,2$, which satisfy the requirements of Theorem 4.6.

One can use the bi-invariant 3 -form $\mathscr{R} e\{$ trace $A B C\}$ to show that $\mathbf{S U}(2)$ is homologically area minimizing in $\mathbf{S U}(n)[\mathbf{T}]$.

5.3. THEOREM. Let $1 \leqslant k_{1}<k_{2}<\cdots<k_{\nu} \leqslant n$ be integers and embed

$$
N=\mathbf{S O}\left(k_{1}\right) \times \mathbf{S O}\left(k_{2}-k_{1}\right) \times \cdots \times \mathbf{S O}\left(k_{\nu}-k_{\nu-1}\right)
$$

as a subgroup of $\mathbf{U}(n)$ is one of the standard ways. Then $N$ is stable.

The proof is analogous to that of Theorem 5.1.

REMARK. It is shown in [T] that $\mathbf{S O}(3)$ is homologically area minimizing in $\mathbf{S O}(4)$.

5.4. One of the motives for our undertaking the investigation in this paper was the explanation of the following example in terms of the geometry of the normal bundle:

Let $M=\mathbf{S}^{3}=\mathbf{C}^{2} \cap\left\{\left(z_{1}, z_{2}\right):\left|z_{1}\right|^{2}+\left|z_{2}\right|^{2}=1\right\}$ and $G=\mathbf{S}^{1}=\mathbf{C} \cap\{\lambda:|\lambda|=1\}$ acting on $\mathbf{S}^{3}$ by scalar multiplication. Then the orbits of $G$ are great circles (so that $\mathbf{v}=2 \pi)$ and are all not stable.

It is convenient to identify $\mathbf{S}^{3}$ with

$$
\mathbf{S U}(2)=\mathbf{G L}(2, \mathbf{C}) \cap\left\{\left[\begin{array}{cc}
z_{1} & z_{2} \\
-\bar{z}_{2} & \bar{z}_{1}
\end{array}\right]:\left|z_{1}\right|^{2}+\left|z_{2}\right|^{2}=1\right\}
$$

by means of the diffeomorphism which projects $A \in \mathbf{S U}(2)$ onto its first row. Thus the metric on $\mathbf{S}^{3}$ corresponds to one-half that on $\mathbf{S U}(2)$.

The action of $G$ on $\mathbf{S U}(2)$ is represented by left multiplication by the one-parameter subgroup $N$ of diagonal matrices $\operatorname{diag}(\lambda, \bar{\lambda}), \lambda \in \mathbf{S}^{1} . D_{12}$ is the unit tangent to $N, I_{12}$ and $J_{12}$ are orthogonal to $N$, and $\left[I_{12}, J_{12}\right]=-2^{-1 / 2} D_{12}$. Thus $\left\|\left[I_{12}, J_{12}\right]\right\| \mathscr{H}^{1}(N)=4 \pi>2 \pi$ and Theorem 4.6 does not apply. 
Returning to $\mathbf{S}^{3}$ we have $N=\mathbf{S}^{1} \times\{0\}$ with unit tangent $X(\lambda, 0)=(i \lambda, 0)$. Normalized vector fields corresponding to $I_{12}, J_{12}$ are respectively $W_{1}(\lambda, 0)=$ $(0,-\lambda), W_{2}(\lambda, 0)=(0, i \lambda) ; W_{12}=-\left[W_{1}, W_{2}\right]=2 X$. A geometrically obvious length decreasing variation of $N$ is given by $W(\lambda, 0)=(0, w)$, where $0 \neq w \in \mathbf{C}$ is fixed. For example, letting $w=-1$ we have $W(\lambda, 0)=\cos \lambda W_{1}+\sin \lambda W_{2}$. It follows from Corollary 3.3 that

$$
\delta^{(2)}(W)=\int_{N}\|d \theta\|^{2}-\langle 2 X, d \theta\rangle d \mathscr{H}^{1}=-2 \pi ;
$$

here $\theta(\lambda, 0)=\lambda$.

The subgroup $N$ of $\mathbf{S U}(2)$ is a maximal torus of $\mathbf{S U}(2)$. More generally, we have the following:

\subsubsection{Theorem. A maximal torus of $\mathbf{S U}(n)$ is not stable.}

Proof. We consider the maximal torus $T$ consisting of the set of diagonal matrices with $\alpha$ th entry $\exp i \lambda_{\alpha}$, where $\lambda_{1}+\cdots+\lambda_{n}=0$. We also embed $\mathbf{S U}(2)$ in $\mathbf{S U}(n)$ in the usual way so that $\mathbf{T}_{I}(\mathbf{S U}(2))$ is spanned by $\left\{I_{12}(I), J_{12}(I), D_{12}(I)\right\} \subset \mathbf{T}_{I}(\mathbf{S U}(n))$.

Defining $N$ and $\theta: N \rightarrow \mathbf{S}^{1}$ as above, $\theta(\operatorname{diag}(\lambda, \bar{\lambda}))=\lambda$, we use the product structure of $T$ to extend $\theta$ smoothly to $T$ so that $\left\langle D_{\gamma, \gamma+1}, d \theta\right\rangle=0$ for $\gamma>1$; define $W=\cos \theta I_{12}+\sin \theta J_{12}$. Clearly, $\|d \theta\|=\|d \theta \mid \mathscr{P}\|$ where $\mathscr{P}$ is the 2-dimensional distribution spanned by $\left\{D_{12}, D_{23}\right\}$, and $\|d \theta \mid \mathscr{P}\|^{2}=\frac{4}{3}\left\langle D_{12}, d \theta\right\rangle^{2}=\frac{2}{3}$ because $\left\langle D_{23}, d \theta\right\rangle=0$. Using Corollary 3.3 with $W_{1}=I_{12}, W_{2}=J_{12}, W_{12}=2^{1 / 2} D_{12}$ we conclude that

$$
\delta^{(2)}(W)=\int_{T}\|d \theta\|^{2}-2^{1 / 2}\left\langle D_{12}, d \theta\right\rangle d \mathscr{H}^{n-1}=-\frac{1}{3} \mathscr{H}^{n-1}(T) .
$$

Finally, we discuss a simple example illustrating Theorem 4.9 which also demonstrates the need for the factor $\left(\nu_{i} \nu_{k}\right)^{1 / 2}$ in Theorem 4.5. Let $N$ denote the oneparameter subgroup $\exp t I_{12}$ of $\mathbf{S U}(2) \subset \mathbf{U}(2)$, acting on $\mathbf{U}(2)$ by left multiplication, and for $c>0$ define the $N$-invariant metric $\sigma_{c}=\sigma_{h}+c \sigma_{v}$ on $\mathbf{U}(2)$ as in 4.9 .

5.4.2. THEOREM. If $c \leqslant \frac{1}{2}$, then $N$ is stable in $\mathbf{U}(2)$; if $c>\frac{1}{2}$, then $N$ is not stable in SU(2).

Proof. Let $W_{1}=J_{12}, W_{2}=D_{12}, W_{3}=2^{-1 / 2}\left(D_{1}+D_{2}\right)$. Then $W_{13}=W_{23}=0$, $W_{12}=2^{1 / 2} I_{12}$. Assume $c>\frac{1}{2}$. Define $\theta: N \rightarrow \mathbf{S}^{1}$ so that $\theta\left(\exp t W_{12}\right)=e^{i t}$ and $W=\cos \theta W_{1}+\sin \theta W_{2}$. Note that $W$ is tangent to $\mathbf{S U}(2)$. Denoting the norms with respect to $\sigma_{c}$ by \|\|$_{c}$ we have $\left\|I_{12}\right\|_{c}=c^{1 / 2}, \mathscr{H}_{c}^{1}(N)=2 \pi(2 c)^{1 / 2}$ and, since $\left\langle I_{12}, d \theta\right\rangle=2^{-1 / 2},\|d \theta\|_{c}=(2 c)^{-1 / 2}$. Applying Corollary 3.3 we obtain $\delta^{(2)}(W)=$ $\left((2 c)^{-1}-1\right) 2 \pi(2 c)^{1 / 2}<0$.

Conversely, if $c \leqslant \frac{1}{2}$, then applying Theorem 4.5 with $X=c^{-1 / 2} I_{12}$ and $\nu_{1}=\nu_{2}$ $=1, \nu_{3}=0$ we have $\left|\left\langle W_{12}, X\right\rangle_{c}\right|=(2 c)^{1 / 2} \leqslant(2 c)^{-1 / 2}=2 \pi / \mathscr{H}_{c}^{1}(N)$, hence $N$ is stable.

On the other hand, if we define $W_{2}=D_{1}$ and $W_{3}=D_{2}$, then $W_{23}=0$ and $W_{12}=I_{12}=-W_{13}$. Seeking to apply Theorem 4.5 with $X$ as above, $\nu_{1}=2$ and $\nu_{2}=\nu_{3}=1$ we have $\left(\nu_{1} \nu_{2}\right)^{1 / 2}\left|\left\langle W_{12}, X\right\rangle_{c}\right|=\left(\nu_{1} \nu_{3}\right)^{1 / 2}\left|\left\langle W_{13}, X\right\rangle_{c}\right|=(2 c)^{1 / 2}$, which is not greater than $2 \pi / \mathscr{H}^{1}(N)$ if and only if $0<c \leqslant \frac{1}{2}$. 


\section{REFERENCES}

[BD] D. Bindschadler, Invariance of solutions to the oriented Plateau problem of maximal codimension, Trans. Amer. Math. Soc. 261 (1980), 439-462.

[BJ1] J. Brothers, Invariance of solutions to invariant parametric variational problems, Trans. Amer. Math. Soc. 262 (1980), 159-179.

[BJ2] _ Second variation estimates for minimal orbits, Proc. Sympos. Pure Math., vol. 44, Amer. Math. Soc., Providence, R.I., 1985.

[F] H. Federer, Geometric measure theory, Springer-Verlag, Berlin and New York, 1969.

[H] W. Y. Hsiang, On compact homogeneous minimal submanifolds, Proc. Nat. Acad. Sci. U.S.A. 56 (1966), 5-6.

[HL] W. Y. Hsiang and H. B. Lawson, Jr., Minimal submanifolds of low cohomogeneity, J. Differential Geometry 5 (1971), 1-38.

[KN] S. Kobayashi and K. Nomizu, Foundations of differential geometry, vols. I, II, Interscience, New York, 1963, 1969.

[L] H. B. Lawson, Jr., Lectures on minimal submanifolds, vol. I, Publish or Perish, Berkeley, Calif., 1980.

[LS] H. B. Lawson, Jr. and J. Simons, On stable currents and their application to global problems in real and complex geometry, Ann. of Math. (2) 98 (1973), 427-450.

[ON] B. O'Neill, The fundamental equations of a submersion, Michigan Math. J. 13 (1966), 459-469.

[S] J. Simons, Minimal varieties in Riemannian manifolds, Ann. of Math. (2) 88 (1968), 62-105.

[T] Dao Chong Thi, Minimal real currents on compact Riemannian manifolds, Math. USSR Izv. 11 (1977), 807-820.

DePartment of Mathematics, Indiana University, Bloomington, Indiana 47405 